\title{
SELECCIÓN DE CEPAS NATIVAS DE Rhizobium leguminosarum bv phaseoli EFICIENTES EN FIJACIÓN BIOLÓGICA DE NITRÓGENO EN SUELOS DE COSTA RICA ${ }^{1}$
}

\author{
Floribeth Mora $^{2}$
}

\begin{abstract}
RESUMEN
Selección de cepas nativas de Rhizobium leguminosarum bv phaseoli eficientes en fijación biológica de nitrógeno en suelos de Costa Rica. Se aislaron 131 cepas de R. leguminosarum bv phaseoli de diferentes zonas frijoleras de Costa Rica. Se evaluaron a nivel de invernadero la respuesta a la inoculación de Phaseolus vulgaris var. Negro Huasteco con estas cepas. Se utilizó macetas y suelo tratado con calor. La inoculación de las semillas se realizó aplicando $10 \mathrm{mi}$ de una suspensión de bacterias de $10^{\circ} \mathrm{ucf} / \mathrm{ml}$ aproximadamente. Además un tratamiento testigo absoluto (sin inocular, sin nitrógeno) y un testigo con nitrógeno (+ $140 \mathrm{~kg} \mathrm{~N} / \mathrm{ha})$, fueron incluidos. Las variables medidas fueron el peso seco de los nódulos, peso seco de la parte aérea y concentración de ureídos del área foliar en la etapa de desarrollo R6. Las plantas inoculadas con las cepas CR-455, CR-487 y CRBSA presentaron los mayores valores de peso seco de nódulos. El testigo absoluto presentó el menor peso seco de nódulos. Se observaron diferencias significativas $(\mathrm{P}<0,05)$, entre algunos de los tratamientos y los testigos. Para la variable peso seco foliar; las plantas que obtuvieron los mayores pesos fueron las inoculadas con las cepas CR-482, CR-492, CR-422 y CR-454. Para la variable concentración de ureídos se registraron diferencias significativas $(\mathrm{P}<0,05)$ entre algunos de los tratamientos y los testigos. Las plantas inoculadas con las cepas CR-475, CR-492 y CR-422, CR-487 y CR-477 presentaron las mayores concentraciones de ureídos, fueron las que mostraron la mayor capacidad de fijar nitrógeno atmosférico en el invernadero. Al comparar los resultados obtenidos con los testigos absoluto y nitrogenado, se observó que existe variación en la efectividad o capacidad para fijar nitrógeno atmosférico entre algunas de cepas de Rhizobium, lo cual concuerda con la literatura.
\end{abstract}

\begin{abstract}
Selection of native strains of Rhizobium leguminosarum bv phaseoli efficient in biological nitrogen fixation in Costa Rican soils. One hundred and thirty one strains of $R$. leguminosarum bv phaseoli were isolated from different bean producing zones in Costa Rica. The response of its inoculation to the Huasteco Negro (Phaseolus vulgaris L.) bean variety was evaluated under green house conditions. Pots and heat treated soil were used. The seeds inoculation was conducted using a bacterial suspension of $10^{\circ} \mathrm{ucf} / \mathrm{ml}$, approximately. Besides, an absolute control (without inoculation, without nitrogen) and a control with nitrogen $(+140 \mathrm{~kg} \mathrm{~N} / \mathrm{ha})$ were included. The dry weight of the nodules, dry weight of the aerial part and the ureids concentration of the foliar area at the R6 stage of development were the measured variables. The plants inoculated with the CR-455, CR-487 and CR-BSA strains showed the largest nodules' dry weight values. The absolute control showed the lowest dry weight of nodules. Significant differences $(\mathrm{P}<0.05)$ were observed among so me treatments and the controls. The plants showing the highest foliar dry weight were those inoculated with the CR-482, CR-492, CR422 and CR-454 strains. Significant differences $(\mathrm{P}<0.05)$ were registered for the ureids concentrations among some treatments and the controls. The plants inoculated with the CR-475, CR492 and CR-422, CR-487 and CR-477 strains had the highest ureids concentrations and also showed the largest atmospheric nitrogen fixing capacity under green house conditions. A variation of the atmospheric nitrogen fixing capacity or effectiveness among some Rhizobium strains was observed, when comparing the results with the absolute and nitrogenous controls, which agrees with the bibliography.
\end{abstract}

\section{INTRODUCCIÓN}

El frijol común (Phaseolus vulgaris L.) es una fuente importante de proteína y calorías para el consumo hu- mano en países tropicales y subtropicales de América Latina (Debouck e Hidalgo, 1985). En Costa Rica es cultivado principalmente por pequeños agricultores en suelos de baja fertilidad y con contenidos subóptimos de

\footnotetext{
1 Presentado en la XL Reunión Anual del PCCMCA en Costa Rica, América Central. 13 al19 de marzo, 1994.

2 Profesora Investigadora de Fitopatología. Universidad Nacional. Heredia, Costa Rica. Apartado Postal 86-3000.
} 
nitrógeno y fósforo. La reducida fertilidad del suelo así como el alto costo o la escasez de fertilizantes contribuyen a los bajos rendimientos observados (Rosas, 1983; Rosas y Bliss, 1986).

Una alternativa para mejorar los rendimientos en este cultivo, es la utilización de inoculantes con rhizobios para incrementar la fijación biológica de nitrógeno. El frijol hasta hace poco era conocido como una de las leguminosas pobres en el establecimiento de una simbiosis efectiva y en su capacidad de fijación de $\mathrm{Nz}$ atmosférico, debido principalmente a la presencia de cepas nativas con poca eficiencia y altamente competitivas en el proceso de infección; sin embargo, hoy día se ha determinado que existe potencial para el incremento de la fijación de nitrógeno en este cultivo mediante una adecuada combinación entre cepas y cultivares (Graham, 1981; Rosas, 1983; Han, 1981; Piha y Mumns 1987; Zamora y Acuña, 1991). Estos últimos autores sugieren inoculaciones con cepas seleccionadas que sean efectivas y competitivas.

Las bacterias pertenecientes al género Rhizobium son capaces de fijar $\mathrm{N}$ en simbiosis con leguminosas, debido a una compleja interacción entre la planta, los rizobios y el ambiente. Para su utilización se debe contar con cepas que presenten competencia saprofítica con otros microorganismos del suelo, competencia con otras cepas de Rhizobium por puntos de infección sobre las raíces y alta eficiencia en fijación de N (Silvester et al., 1987; Zamora y Acuña, 1991).

Una cepa es efectiva (habilidad para fijar N) cuando ésta presenta infectividad (habilidad para infectar las raíces de las plantas), persiste y crece en el suelo, tolera las condiciones locales, compite por sitios de infección con otros organismos de la rizosfera $\mathrm{y}$, manifiesta estabilidad genética (Silvester et al., 1987).

Rosas, (1986), Piha y Munns (1987) han demostrado que, entre un 25 y un $75 \%$ del $\mathrm{N}$ requerido por la planta de frijol, proviene de la fijación simbiótica. Las cantidades de $\mathrm{N}_{2}$ fijado suministran entre $25-125 \mathrm{~kg}$ $\mathrm{N} / \mathrm{ha}$, dependiendo del cultivar.

Uribe et al. $(1987,1990)$ determinaron que no hubo un aumento significativo en la producción de frijol, cuando éste fue inoculado con rhizobios introducidos de otros países. En las zonas de Monterrey de San Carlos y San Pedro de Pérez Zeledón, Costa Rica, donde no se obtuvo respuesta significativa a la inoculación, el comportamiento de los tratamientos testigos fue aceptable. Estos resultados que podrían indicar la presencia de cepas nativas efectivas. En general el efecto observado, con la inoculación de cepas introducidas puede atribuirse a tres razones principales: i) las condiciones ambientales y edáficas en nuestro medio difieren de las que imperan en el lugar donde las cepas fueron aisladas; ii) la diferente capacidad de nodulación y fijación entre cepas (Grahan, 1981; Morales, 1987); iii) las poblaciones nativas de $R$. phaseoli que compiten por puntos de infección con las cepas introducidas.

Debido a lo expuesto anteriormente, se deduce que es necesario contar con cepas efectivas y competitivas en la fijación biológica de $\mathrm{N}$ (FBN), seleccionadas de una amplia variedad de aislamientos obtenidos de diferentes lugares; para la elaboración de inoculantes que puedan ser utilizados por los agricultores en plantaciones comerciales de frijol.

Como criterio de selección se pueden utilizar varios parámetros: i) peso seco de la parte aérea de las plantas en la etapa de floración (R6) (Debouck e Hidalgo, 1985); ii) incremento en la masa, peso seco, distribución, color y número de nódulos por planta, en la misma etapa (Somasegaran et al., 1981 ).

La evaluación exacta de la actividad de los nódulos en la fijación biológica, por medio de la enzima nitrogenasa, puede lograrse a traves de varios métodos: a) reducción del acetileno, donde se determina la producción de etileno e indirectamente la actividad en la fijación; b) determinación de la acumulación total de $\mathrm{N}$ en la parte 
aérea por el método de microkjeldhal, y c) determinación de ureídos (Somasegaran et al., 1981; Silvester et al., 1987; Castro, 1992).

El presente experimento tuvo como objetivo aislar y seleccionar cepas nativas de Rhizobium leguminosarum biovar phaseoli que sean eficientes en la fijación biológica de nitrógeno, de diversas localidades de Costa Rica.

\section{MATERIALES Y MÉTODOS}

Muestreo y aislamiento Rhizobium leguminosarum bv phaseoli

Los muestreos de nódulos de frijol fueron realizados durante agosto, setiembre y octubre de 1991, en las zonas de Upala, San Carlos, Los Chiles, Atenas, Esparza, San Ramón, Puriscal, Cartago, Pérez Zeledón, Buenos Aires, Coto Bms y Acosta.

Los muestreos se realizaron en forma casual en pequeñas plantaciones de frijol, que estuvieran en la etapa R6 de desarrollo fisiológico (Debouck e Hidalgo, 1985). Los agricultores utilizaban diferentes variedades de frijol (Parrilla, Huetar, Negro Huasteco, Chimbolo, Criollo, etc.). Se constató que estos agricultores no habían inoculado con rhizobios ni fertilizado a la siembra. Los nódulos de una planta fueron colectados en un tubo de ensayo con sílica gel (Acuña, 1991; Somasegaran, Hoben y Halliday, 1981).

Posteriormente los rhizobios fueron aislados $\mathrm{y}$ purificados mediante la metodología descrita por Somasegaran et al. (1981).

\section{Evaluación de efectividad de las cepas aisladas}

Los experimentos fueron realizados a partir de noviembre de 1991, en los invernaderos y laboratorios del
Centro de Investigaciones Agronómicas de la Universidad de Costa Rica.

Debido al alto número de cepas aisladas (131), fue necesario realizar evaluaciones preliminares a fin de seleccionar las cepas de comportamiento superior. Con esta finalidad se dividieron en tres diferentes gmpos, y se realizaron tres experimentos utilizando la siguiente metodología:

1) Muestras de $2,5 \mathrm{~kg}$ de una mezcla de suelo-arena (2: 1), tratadas con calor $\left(3 \mathrm{~h} \mathrm{a} 120^{\circ} \mathrm{C}\right.$ y 1 atmósfera de presión), fueron colocadas en macetas de plástico. Las características del suelo se muestran en el Cuadro 1.

2) En cada maceta se aplicó $0,15 \mathrm{~g}$ de $\mathrm{N}$ (fuente $\mathrm{NH}_{4} \mathrm{Cl}$ ) como fertilización básica para favorecer el establecimiento de las cepas en el cultivo.

3) Cinco semillas de frijol variedad Negro Huasteco, fueron sembradas por maceta. La inoculación se realizó asperjando $10 \mathrm{mi}$ de una suspensión de $10^{9}$ ucf/ml (aproximadamente), de cada una de las cepas de $R$. leguminosarum biovar phaseoli, preparada a partir de cultivos puros aislados en el medio de cultivo agar-levadura-manitol (Somasegaran, Hoben y Halliday, 1981). La inoculación se realizó antes de colocar el suelo sobre las semillas. Transcurridos los 22 días se procedió a ralear a tres plantas/maceta.

4) Los tratamientos evaluados fueron: Las cepas de R. leguminosarum biovar phaseoli, un testigo relativo (+0,72 $\mathrm{g}$ de $\mathrm{NH}_{4} \mathrm{Cl} /$ maceta, de nitrógeno, equivalente a $140 \mathrm{~kg} \mathrm{~N} / \mathrm{ha}$ ) y un testigo absoluto (sin inocular, sin adicionar $\mathrm{N}$ ).

Se utilizó un diseño irrestricto al azar con cuatro repeticiones por tratamiento. Cada unidad experimental consistió de cuatro macetas con tres plantas cada una.

Cuadro 1. Análisis químico del suelo utilizado en la evaluación de efectividad de las cepas aisladas.

\begin{tabular}{|c|c|c|c|c|c|c|c|c|c|c|c|c|c|}
\hline $\begin{array}{l}\text { pH } \\
\mathbf{H}^{2} \mathbf{0}\end{array}$ & $\begin{array}{c}\text { M.O. } \\
\%\end{array}$ & $\frac{\mathrm{Ca}}{\mathrm{cI}}$ & $\frac{\mathrm{Mg}}{\mathrm{lol}(+}$ & $\mathbf{K}$ & Acidez & CICE & $\mathbf{P}$ & $\mathrm{Zn}$ & $\frac{\mathrm{Mn}}{\mathrm{m} / \mathrm{L}}$ & $\mathrm{Cu}$ & $\mathrm{Fe}$ & B & $\mathbf{S}$ \\
\hline 6 & 10,38 & 11,8 & 2,9 & 1,69 & 0,3 & 16,69 & 77 & 52 & 9 & 18 & 384 & 2 & 80 \\
\hline
\end{tabular}


5) Las variables dependientes medidas fueron:

a- Peso seco de parte aérea/maceta

b- Peso seco de nódulos/maceta

c- Contenido de ureídos de parte aérea/maceta, determinado con el método descrito por Vogeles et al. (1970) y Paterson et al. (1983).

Las evaluaciones se realizaron en la etapa de crecimiento R6 (Debouck e Hidalgo, 1985). Cada experimento se le practicó el análisis de varianza y pruebas de Duncan al 0,05 para la separación de medias.

6) Se realizó un cuarto experimento, para lo cual se utilizó el 10\% de las cepas más efectivas en cada uno de los tres experimentos anteriores (según la prueba de Duncan $\mathrm{P}<0,05)$.

En este último experimento se evaluaron 18 cepas (Cuadro 2) más los tratamientos testigos $(+\mathrm{N}$ y $\sin$

Cuadro 2. Procedencia de las cepas de Rhizobium leguminosarum bv phaseoli, seleccionadas como más efectivas en las evaluaciones preliminares.

\begin{tabular}{ll}
\hline Cepa & \multicolumn{1}{c}{ Procedencia } \\
\hline CR-475 & Cervantes, Cartago \\
CR-492 & Polka, Puriscal \\
CR-422 & San Juan, San Ramón \\
CR-487 & Pedernal, Puriscal \\
CR-477 & Cervantes, Cartago \\
CR-BSA & San Antonio Belén, Heredia \\
CR-455 & Los Chiles, San Carlos \\
CR-488 & Pedernal, Puriscal \\
CR-436 & Polka, Puriscal \\
CR-482 & Santiago, Puriscal \\
CR-4009 & El Coco, Alajuela \\
CR-4025 & San Juan, San Ramón \\
CR-479 & Cervantes, Cartago \\
CR-442 & Santa Rosa, Cutris, San Carlos \\
CR-432 & Paraíso, Cartago \\
CR-416 & Polka, Puriscal \\
CR-454 & Los Chiles, San Carlos \\
CIAT-632 & CIAT, Colombia \\
\hline
\end{tabular}

inocular). Se utilizó la metodolgía descrita anteriormente y se evaluaron las mismas variables.

\section{RESULTADOS Y DISCUSIÓN}

En el muestreo que se realizó en las diferentes zonas frijoleras a nivel nacional fueron aisladas 131 cepas de $R$. leguminosarum biovar phaseoli, lo que demuestra que la población nativa de estos microorganismos en nuestros suelos es abundante; estos resultados concuerdan con las observaciones realizadas por Soto (1992); no obstante, al no conocerse su variabilidad no se puede afirmar que estos aislamientos sean diferentes entre sí.

La variabilidad genética de las cepas, se puede manifestar por la diferencia que éstas presentan en la habilidad competitiva por sitios de formación de nódulos, la cual está determinada por la interacción del genoma de la planta, de la cepa introducida: de la población nativa y de los fac tores ambientales. Debido a que la respuesta a la inoculación y éxito competitivo de cada uno de los aislamientos es desconocido, es necesario evaluar su capacidad de fijación primero a nivel de invernadero $y$, posteriormente, a través de parcelas de validación a nivel de campo, antes de dar una recomendación final al agricultor (Silvester et al., 1987).

En lo que respecta al experimento No. 4, en la variable peso seco de nódulos la mayor respuesta fue obtenida con la inoculación de las cepas CR-455, CR487, CR-BSA y CR-492, los cuales mostraron diferencias significativas con respecto a otros tratamientos (Cuadro 3). El testigo absoluto presentó el menor peso seco de nódulos; la formación de nódulos en este tratamiento, así como en el testigo $+\mathrm{N}$, se debió, posiblemente, a que en el suelo se encontraban rhizobios viables capaces de infectar las raíces, a pesar de que fue tratado con calor con el objetivo de disminuir al mínimo la población de cepas nativas; es decir, no se puede asegurar que el suelo estuviera totalmente estéril.

La habilidad de las cepas para formar nódulos, no necesariamente indica la eficiencia en la FBN. Para cada 
Cuadro 3. Efecto de las primeras cepas seleccionadas de Rhizobium leguminosarum biovar phaseoli, y de la aplicación de N, sobre el peso seco foliar, peso seco de nódulos y concentración de ureídos en plantas de frijol en la etapa R6, en condiciones de invernadero.

\begin{tabular}{|c|c|c|c|c|c|}
\hline Trat. & $\begin{array}{l}\text { Peso seco de } \\
\text { nódulos (g) }\end{array}$ & Tratamiento & Peso seco foliar (g) & Tratamiento & $\begin{array}{l}\text { Concentración } \\
\text { de ureídos (ug/g) }\end{array}$ \\
\hline CR-455* & $0,4460 a^{* *}$ & CR-482 & $15,21 \mathrm{a}$ & CR-475 & $231,5 \mathrm{a}$ \\
\hline CR-487 & $0,3181 \mathrm{ab}$ & CR-492 & $15,01 \mathrm{ab}$ & CR-492 & $227,4 a$ \\
\hline CR-BSA & $0,2989 a b c$ & CR-422 & $14,72 \mathrm{ab}$ & CR-422 & $222,2 \mathrm{ab}$ \\
\hline CR-492 & $0,2970 \mathrm{abc}$ & CR-454 & $13,28 \mathrm{abc}$ & CR-487 & $215,0 a b c$ \\
\hline CR-475 & $0,2937 \mathrm{abcd}$ & CR-479 & $13,06 \mathrm{abc}$ & CIAT-632 & $198, \mathrm{abc}$ \\
\hline CR-422 & 0,2726 abcde & CR-487 & $12,72 \mathrm{abc}$ & CR-477 & $186,3 \mathrm{abc}$ \\
\hline CR-482 & 0,2501 bcde & CR-432 & $12,65 \mathrm{abcd}$ & CR-BSA & 179,4abcd \\
\hline CR-432 & 0,2398 bcde & CR-475 & $12,41 \mathrm{abcd}$ & CR-455 & $155,6 \mathrm{bcde}$ \\
\hline CR-479 & 0,2039 bcde & CR-400 & $12,19 \mathrm{bcde}$ & CR-488 & $153,8 \mathrm{bcde}$ \\
\hline CR-454 & 0,22033 bcde & CR-4025 & $11,57 \mathrm{cde}$ & CR-436 & $149,9 \mathrm{cdef}$ \\
\hline CR-416 & 0,2008 bcde & $+\mathrm{N}$ & $11,52 \mathrm{cde}$ & CR-482 & 114,5 defg \\
\hline CIAT-632 & $0,1990 \mathrm{bcde}$ & CR-455 & $11,44 \mathrm{cde}$ & CR-4009 & $105,3 \mathrm{efgh}$ \\
\hline CR-477 & $0,1958 \mathrm{bcde}$ & CIAT-632 & $11,02 \mathrm{cde}$ & CR-4025 & $102,9 \mathrm{efgh}$ \\
\hline$+\mathrm{N}$ & $0,1882 \mathrm{bcde}$ & CR-436 & $10,90 \mathrm{cde}$ & CR-479 & 92,0 efgh \\
\hline CR-436 & $0,1419 \mathrm{cdef}$ & CR-477 & $10,62 \mathrm{cde}$ & CR-442 & 90,4 efgh \\
\hline CR-442 & $0,1260 \mathrm{def}$ & CR-416 & $10,34 \mathrm{cde}$ & $+\mathrm{N}$ & 79,3 fgh \\
\hline CR-488 & 0,1185 ef & CR-442 & $10,05 \mathrm{de}$ & CR-432 & $65,8 \mathrm{gh}$ \\
\hline CR-4009 & $0,008750 \mathrm{f}$ & CR-488 & $9,99 \mathrm{de}$ & CR-416 & $60,6 \mathrm{gh}$ \\
\hline CR-4025 & $0,000100 \mathrm{f}$ & CR-BSA & $9,78 \mathrm{de}$ & $\mathrm{TA}$ & $57,3 \mathrm{gh}$ \\
\hline $\mathrm{TA}$ & $0,000100 \mathrm{f}$ & $\mathrm{TA}$ & $9,06 \mathrm{e}$ & CR-454 & $35,7 \mathrm{~h}$ \\
\hline
\end{tabular}

* C. R.= Costa Rica (sinominia bacterioteca Microbiología de Suelos-CIA-UCR).

** Letras diferentes en una misma columna indican diferencias significativas según la prueba de Duncan.

combinación leguminosa-rhizobio, el nivel óptimo de nodulación es diferente. No obstante, los parámetros de abundancia, tamaño, distribución y coloración interna de los nódulos son importantes indicadores de su efectividad o habilidad para fijar nitrógeno gaseoso (Silvester et al., 1987). Es común observar leguminosas "promiscuas" que presentan una nodulación "semiefectiva" en donde se observa una buena nodulación, pero fijan una reducida cantidad de N, que no les permite alcanzar su potencial de rendimiento. De esta manera, no todas las cepas de Rhizobium son efectivas en la fijación de nitrógeno, a pesar de que la infectividad sea alta (Mumns, 1987).
El peso seco sirvió para determinar la influencia de los tratamientos sobre la producción de materia verde. Se observaron diferencias significativas $(\mathrm{P}<0,05)$ entre los tratamientos y el testigo absoluto (Cuadro 3). Los valores más altos fueron obtenidos cuando se inocularon las cepas CR-482, CR-492, CR-422 y CR-454; las cuales superaron el peso seco foliar del testigo nitrogenado, aunque sólo en las tres primeras se presentaron diferencias significativas con éste. Los menores valores los presentaron las plantas inoculadasconlacepa CR-BSA y el testigo absoluto, tratamientos en los que, además, se observó un reducido desarrollo foliar. 
Los resultados anteriores indican que al menos $10 \mathrm{de}$ las cepas evaluadas fueron capaces de suministrar tanto $\mathrm{N}$ como lo hizo el testigo relativo $(140 \mathrm{~kg} / \mathrm{ha})$, pues con todos estos tratamientos se obtuvo un crecimiento y desarrollo foliar similar de las plantas en invernadero, donde la competencia con cepas nativas es mínima, (debido al aumento del nivel poblacional de las cepas inoculadas y a que el suelo fue tratado), como si es posible que se presente en el campo, bajo las condiciones climáticas, edáficas y ecológicas que prevalecen en Costa Rica (Acuña, 1991; Soto, 1992). Es importante anotar que la disponibilidad de $\mathrm{N}$ proveniente de FBN se inicia aproximadamente 20 días después de la siembra, debido a lo cual se hace necesario adicionar una pequeña cantidad de $\mathrm{N}$ mineral ( $30 \mathrm{~kg} \mathrm{~N} / \mathrm{ha}$ ), para estimular el crecimiento inicial de las plántulas, favorecer el establecimiento de las cepas en el cultivo y la formación de nódulos (Silvester, 1983).

En el Cuadro 3 se observa que las mayores concentraciones de ureídos se obtuvieron en las plantas que fueron inoculadas con las cepas CR-475, CR-492, CR-422 y CR-487. Se detectaron diferencias significativas $(\mathrm{P}<0,05)$ al comparar sus respuestas con otros tratamientos incluyendo los testigos. Según McClure e Israel (1979) y Sprent (1980) los ureídos transportan el 90\% del nitrógeno fijado en los nódulos de plantas completamente dependientes de la simbiosis; este elemento es transportado principalmente como ami das (asparagina y ácido alantóico), que están presentes sólo en pequeñas cantidades en plantas dependientes del $\mathrm{N}$ mineral. Estas observaciones sugieren que la abundancia de ureídos puede reflejar la dependencia simbiótica de las plantas y proveer una medida cuantitativa de la fijación de $\mathrm{N}$ atmosférico (Castro, 1992). En concordancia con lo expuesto anteriormente, en este experimento uno de los contenidos más bajos de ureídos se presentó en el testigo absoluto; un nivel también muy bajo se obtuvo con la cepa CR-454, a pesar de presentar alto peso seco foliar, lo cual se puede deber a una alta eficiencia asimilatoria de los nutrimentos extraídos del suelo por parte de la planta, cuando ésta es infectada por Rhizobium, debido a lo cual no le es necesario fijar nitrógeno atmosférico.
Tanto el testigo nitrogenado como el testigo absoluto presentaron nódulos y contenido de ureídos. A pesar de que estos valores fueron bajos, esto evidencia que en el suelo utilizado habían rhizobios viables, aunque no se descartan otras posibles fuentes de contaminación (salpique por riego, salpique por pipetas, etc.). Mc Clure e Israel (1979) determinaron, en experimentos con plantas de soya noduladas y no noduladas, que la mayor cantidad de la síntesis de ureídos está asociada con la presencia de nódulos; sin embargo, existen bajos niveles de ureídos en la savia del xilema de plantas no inoculadas, lo que sugiere que alguna proporción de ureídos se produce aún cuando los nódulos no están presentes.

Al evaluarse el comportamiento de las 18 cepas bacterianas en las diferentes variables analizadas, se obtuvieron resultados que evidencian que existe variabilidad en la habilidad de los nódulos para fijar nitrógeno. La cepa de R. leguminosarum bv phaseoli que se utilice como inoculante, deberá ser seleccionada de una amplia variedad de cepas. Esta selección constituye la primera y fundamental etapa en el proceso de elaboración de inoculantes para leguminosas en cultivos comerciales (Silvester et al., 1987). Con el propósito de dar seguimiento a esta investigación, se recomienda evaluar las cepas CR-475, CR-492, CR-422, CR-487 y CR-477 a nivel de campo en los próximos experimentos. Las primeras cuatro presentaron los mayores contenidos de ureídos, peso seco foliar y peso seco de nódulos; la cepa CR-477 fue seleccionada porque presentó alto contenido de ureídos.

Es importante evaluar la capacidad competitiva por puntos de infección, que puedan presentar estas cepas con la población nativa de Rhizobium presente en el suelo, cuando se trabaja directamente en el campo.

\section{LITERATURA CITADA}

ACUÑA, O. 1991. Manejo y tecnología en la asociación simbiótica Rhizobium-Ieguminosa. In: Curso de Microbiología de Suelos. San José, Universidad de Costa Rica, Centro de Investigaciones Agronómicas. 4p. 
CASTRO, L.; ACUÑA, O. 1992. Determinación de la concentración de ureídos en tres leguminosas fijadoras de nitrógeno: soya, frijol y maní. Agronomía Costarricense 16(2): 187-193.

DEBOUCK, D.; HIDALGO, R. 1985. Morfología de la planta de frijol común. In Frijol: Investigación y producción. Ed. por M. López, F. Fernández y A. Schoonhoven. Cali, Colombia, CIAT - PNUD. p.7-42.

GRAHAM, P. 1981. Some problems of nodulation and symbiotic nitrogen fixation in Phaseolus vulgaris L: a review. Field Crops Research 4: 93-112.

HAN, P. 1981. Some problems of nodulation and symbiotic nitrogen fixation in common bean. Agronomy Journal 71(6):925-926.

McCLURE, P.; ISRAEL, D. 1979. Transport of nitrogen in the xilem of soybean plant. Plant Physiology 64:411-416.

MORALES, M. 1987. Selección y evaluación de cepas de Rhizobium leguminosarum biovar phaseoli tolerantes al suministro restringido de fósforo. Tesis Mag. Sc. Turrialba, Costa Rica, UCR/CATIE. 97p.

MUMNS, D.N. 1987. Nitrogen fixation potential of bean $(P$. vulgaris) compared with other grains legumes under controlled conditions. Plant and Soil 98(2): 169-182.

PIHA,M.; MUMNS,D. 1987. Sensitivityofthecommon bean ( $P$. vulgaris) symbiosis to high soil temperature. Plant and Soil 98(2): 183-194.

ROSAS, J. 1983. Principios y prácticas para la conducción de ensayos sobre fijación de nitrógeno en condiciones de campo. Ceiba 27(1):23-39.

ROSAS, J.; BLISS, F. 1986. Utilización del potencial de fijación de nitrógeno del frijol común en Centro América. Ceiba 27: 105-115.

SILVESTER, R.; KIPE, J.; HARRIS, D. 1987. Simbiosis leguminosa-rizobio: Evaluación, selección y manejo. Centro Internacional de Agricultura Tropical. Cali, Colombia. $72 \mathrm{p}$.
SILVESTER, R. 1983. Fijación biológica de nitrógeno por leguminosas: aspectos agronómicos relacionados con su nodulación con Rhizobium. Suelos Ecuatoriales 13(2):2835 .

SOMASEGARAN, P.; HOBEN, H.; HALLIDA Y, J. 1981. Ejercicios prácticos en tecnología Rhizobium-Ieguminosa. Curso regional de entrenamientos sobre la simbiosis entre Rhizobium y leguminosas tropicales de importancia agrícola. Centro de Investigaciones Agronómicas, Facultad de Agronomía. Universidad de Costa Rica. 85 p.

SOTO, G. 1992. Geographic distribution and enviromental interactions that affect the abundance of Rhizobium leguminosarum biovar phaseoli in Costa Rica. M.Sc. Thesis Department of Environmental Sciences, University of Virginia. 207p .

SPRENT, J. 1980. Root nodule anatomy, type of export product and evolutionary origen of so me leguminosae. Plant Cell Environ. 3:35-43.

URIBE, L.; ACUÑA, O.; HERNANDEZ, G. 1990. Respuesta de Phaseolus vulgaris var. Negro Huasteco a la inoculación con tres cepas de Rhizobium bajo condiciones de mínima labranza en tres localidades de Costa Rica. Agronomía Costarricense 14(2):201-206.

URIBE, L.; ACUÑA, O. 1987. Efecto de la fertilización y tipo de suelo sobre la inoculación con diferentes cepas de Rhizobium leguminosarum biovar phaseoli en frijol común. In: Primera Reunión Centroamericana de Fijación Biológica de Nitrógeno. Guatemala. 67 p.

VOGELES, G. VAN DER DRIFT. 1970. Differential analyses of glyoxylate derivatives. Analytical Biochemistry 33: 143-157.

ZAMORA, A.; ACUÑA, O. 1991. Mejoramiento de la fijación biológica de nitrógeno en el frijol común. In: Proyectos Regionales de Investigación. Informes anuales. Profrijol. 369 p.

ZAMORA, A.; ACUÑA, O.; RODRIGUEZ, J. 1990. Mejoramiento de la fijación biológica del nitrógeno en el frijol. In: Proyectos Regionales de Investigación. Planes de trabajo 1990-1992. Profrijol. 173 p. 